\title{
A Rare Case of Extramedullary Multiple Myeloma Presenting as Masses on the Breast and Chest Wall Complicated with Sjogren's Syndrome
}

This article was published in the following Dove Press journal:

International Journal of General Medicine

\author{
Hongmei Yuan ${ }^{1,2}$ \\ Huilan Lei ${ }^{2}$ \\ Peng $\mathrm{Gu}^{2}$ \\ Lang $\mathrm{Ma}^{\prime}$ \\ Li Qiu'
}

'Department of Medical Ultrasound, Laboratory of Ultrasound Imaging Drug, West China Hospital, Sichuan University, Chengdu 61004I, People's Republic of China; ${ }^{2}$ Department of Ultrasound, Sichuan Key Laboratory of Medical Imaging, Affiliated Hospital of North Sichuan Medical College, Nanchong 637000, People's Republic of China

Correspondence: Li Qiu

Department of Medical Ultrasound, West China Hospital, Sichuan University, No.

37 Guoxue Alley, Chengdu 61004I,

People's Republic of China

Tel +86- I8980602044

Fax +86-28-85I64I 46

Email qiulihx@scu.edu.cn

\begin{abstract}
Multiple myeloma (MM) in the breast has been rarely documented. However, there are no reports on concurrent masses of chest wall soft tissue and breast as the primary symptoms for MM. Herein, we report a case of a 68-year-old woman presented with masses in her right breast and chest wall for 1 month. She recalled having xerophthalmia and xerostomia for 2 years without taking any test to search for the cause of these symptoms. Ultrasonography (US) and computed tomography (CT) examination revealed masses in the right breast and chest wall. Both lesions of the breast and chest wall were confirmed to be plasma cell tumors by ultrasound-guided puncture biopsy and MM was confirmed by bone marrow examination. Meanwhile, Sjogren's syndrome (SS) was diagnosed by the positive expression of antibodies against antinuclear, Ro/SSA and La/ SSB. After three courses of chemotherapy for MM, the symptoms of SS were significantly alleviated and the masses in the chest wall and breast disappeared.
\end{abstract}

Keywords: multiple myeloma, Sjogren's syndrome, breast neoplasms, chest wall tumors

\section{Introduction}

Myeloma is a malignant tumor originating from plasma cells in the bone marrow, which includes two types, ie solitary plasmacytoma and MM. MM is more common and predominantly occurs in men. It is caused by the malignant transformation of plasma cells, which can synthesize and secrete immunoglobulins. A large number of proliferative monoclonal malignant plasma cells can cause pathological changes in tissues and organs, mainly in the bones, kidneys, blood system, and nervous system. ${ }^{1}$ Previous studies showed that the most common symptoms of MM were fatigue and bone pain, ${ }^{2}$ while masses in the chest wall soft tissue and breasts as the primary symptoms have not been reported. SS is an autoimmune disease, characterized by infiltration of lymphocytes and plasma cells in the exocrine glands. The patients with primary SS had 44 times higher risk of developing B-cell lymphoproliferative diseases, such as benign monoclonal gammopathy, in particular, compared to the normal population. ${ }^{3}$ However, only few studies have demonstrated that $\mathrm{MM}$ is associated with $\mathrm{SS},{ }^{4,5}$ and most MM complicated with SS showed IgM type. Herein, we report a rare case of a female patient with $\operatorname{IgD}$-lambda $(\lambda)$ type MM complicated with SS presenting as masses on the breast and chest wall.

\section{Case Presentation}

A 68-year-old woman presented with a one-month history of masses in the breast and chest wall. The patient recalled having dysphagia for dry food, xerophthalmia, 
xerostomia, and weight loss for 2 years; however, she did not undergo any test to search the cause of these symptoms. Physical examination revealed two masses in the chest wall and right breast, respectively, which showed poor toughness and mobility. On ultrasound examination, a heterogeneous hypoechoic mass $(2.2 \mathrm{~cm} \times 1.2 \mathrm{~cm})$ with irregular shape and margin, angular periphery and incomplete capsule was revealed in the right breast about $5 \mathrm{~cm}$ from the nipple at the 10 o'clock position. The mass showed an aspect ratio less than 1.0 and posterior acoustic enhancement. No obvious blood flow was detected by color Doppler imaging (Figure 1A). It was classified as category 4 (suspicion for malignancy) according to the Breast Imaging Reporting and Data System for ultrasonography. The left breast and both axillary areas had no abnormality. In addition, another heterogeneous hypoechoic mass $(5.8 \mathrm{~cm} \times 2.2 \mathrm{~cm})$ with striped hyperechoic features and unclear demarcation from surrounding muscle was found in the left anterior chest wall (Figure 1B). Striped blood flow with a high resistance index of 0.78 was revealed by color Doppler imaging (Figure 1C). Subsequent CT examination showed a slightly high-density mass with regular shape and margin in the right breast (Figure 2A). Likewise, a soft-tissue density mass was seen in the chest wall around the sternal stalk, and there was no distinct demarcation between the mass and the pectoralis major muscle (Figure 2B). All the masses showed homogeneous enhancement on enhanced CT images (Figure 2C and D). In addition, no significant abnormality was found in the skull, pelvis and hip bones by X-ray findings.

Ultrasound-guided biopsy of breast and chest wall masses was done, and the hematoxylin and eosin (HE) staining of the sections showed lymphocyte proliferation (Figure 3A). Immunohistochemical analysis showed that the neoplastic cells were positive for CD138 (Figure 3B),
$\operatorname{IgD}$ (Figure 3C) and restriction for $\lambda$ light chain, but negative for CD56 (Figure 3D) and CD117 (Figure 3E). The diagnosis based on the pathological examination was plasma cell tumors. However, full blood count, liver and kidney functions were all in normal ranges. And then bone marrow biopsy revealed that the immature plasma cells accounted for 56\% (Figure 3F). Meanwhile, flow cytometry of bone marrow aspirate with CD138/CD38 gating strategy showed positive expression for CD38, CD138, CD10, CD20, CD28, CD33, and $\lambda$. Serum protein electrophoresis and immunofixation were subsequently requested, showing monoclonal (M) protein (9.70\%) with type $\operatorname{IgD} \lambda$ paraprotein $(7.240 \mathrm{~g} / \mathrm{L})$. In addition, urinary Bence Jones protein was positive for $\lambda$ free light chain, which showed kappa $(\kappa)$ and $\lambda$ at a concentration of $0.038 \mathrm{~g} / \mathrm{L}$ and $2.910 \mathrm{~g} / \mathrm{L}$, respectively, the $\kappa / \lambda$ ratio decreased significantly. According to the clinicopathological and laboratory findings, $\mathrm{MM}(\operatorname{IgD} \lambda$, stage III A) was diagnosed. Furthermore, we performed some tests to reveal the cause of xerostomia and dry eyes. Antinuclear antibodies were positive at 1/320, and the count of antibodies against Ro/SSA and La/SSB in serum was more than $200 \mathrm{U} / \mathrm{mL}$. The presence of these antibodies along with clinical symptoms established the diagnosis of SS.

Based on the above findings, the patient was diagnosed as extramedullary MM complicated with primary SS, and then she was treated with bortezomib, thalidomide and dexamethasone. The clinical symptoms were improved after three courses of therapy, xerostomia and xerophthalmia alleviated, and the breast and chest wall masses almost disappeared simultaneously on repeated CT examination (Figure 2E and F). The patient's condition was stable, and we will continue to follow her outcome.
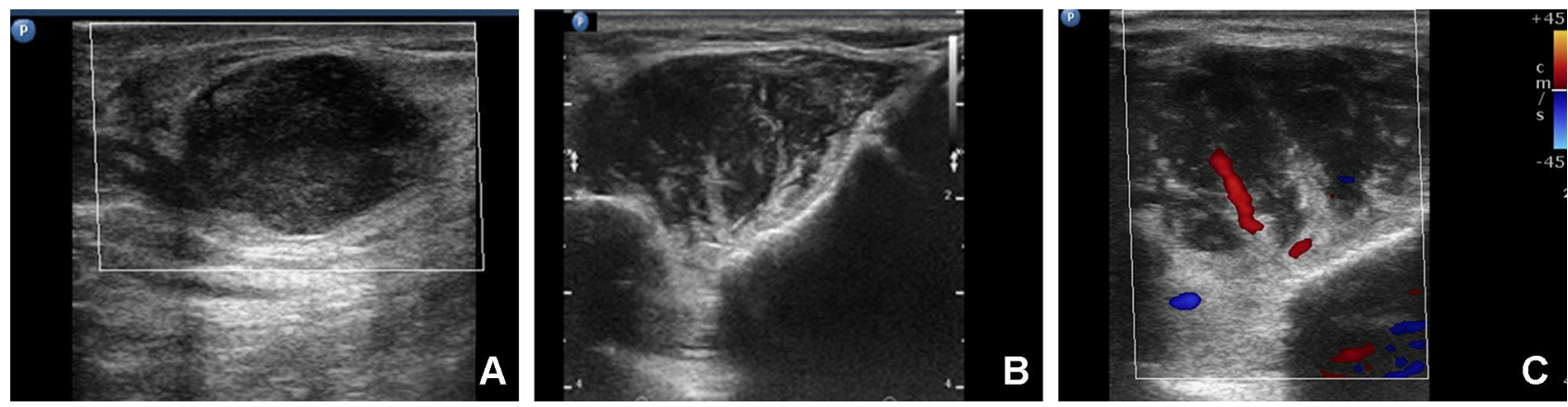

Figure I Ultrasound examination of the masses in the right breast and chest wall. (A) A heterogeneous hypoechoic mass with enhanced echo in the rear was found in the right breast. (B) A hypoechoic mass with striped hyperechoic feature was found in the chest wall. (C) Color Doppler Imaging of the mass in the chest wall displayed strip blood flow with high resistance index. 

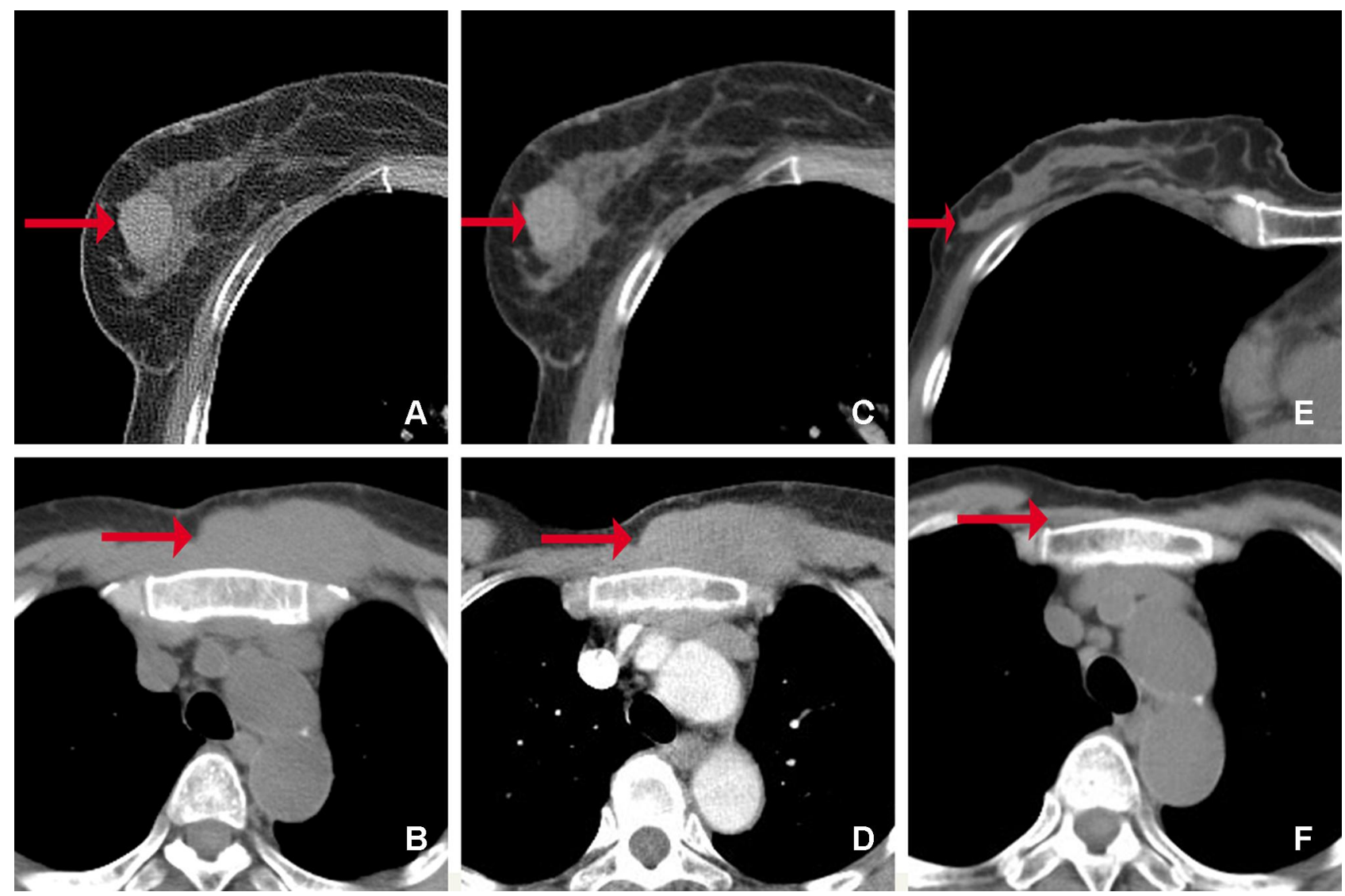

Figure 2 CT images of the masses in the right breast and chest wall. Before treatment (A-D): (A) There was a well-defined slightly high-density mass in the right breast (arrow). (B) An ill-defined iso-density mass is shown in the chest wall (arrow). The masses in the breast (C) and the chest wall (D) show homogeneous and mild enhancement on enhanced CT images (arrow). After treatment (E and $\mathbf{F})$ : the masses of the breast (E) and chest wall (F) disappeared (arrow).

\section{Discussion}

MM is classified as a plasma cell neoplasm by the World Health Organization (WHO), ${ }^{6}$ A large quantity of an abnormal immunoglobulin called $\mathrm{M}$ protein produced by plasma cells derived from B cells is a cardinal feature of $\mathrm{MM}^{7}$ SS was diagnosed according to the criteria of the American College of Rheumatology (ACR) Classification, ${ }^{8}$ it is an autoimmune disease characterized by lymphocytic infiltration of the exocrine glands and a polyclonal B cells activation. ${ }^{3}$ It has been well documented that SS has a higher incidence of B cell lymphomas with high standardized incidence ratio (SIR) of 4.9-44. ${ }^{9,10}$ While only few reported cases have shown that SS may be associated with MM. ${ }^{3,5,11}$ The relationship between MM and SS is still not clear. Some studies have shown that chronic inflammatory stimulation and immune disturbance of SS may be important factors for the development of MM. ${ }^{3}$ A systematic review showed the incidence of MM was associated with autoimmune diseases such as SS for the IgA, IgM, and IgG may induce autoimmunity. ${ }^{12}$ While this case was the rare type $\operatorname{IgD}$ MM which was found in $2.0-2.5 \%$ of the patients. ${ }^{13}$ Studies have reported that IgD myeloma is usually associated with $\lambda$ light chain and has a higher risk of extramedullary infiltration, ${ }^{14}$ which is consistent with our reports. Most of the patients with IgD MM tended to be younger (median age, 52 years) and more likely to have distinct clinical features, such as renal dysfunction, features of advanced disease. ${ }^{13}$ However, this case is a 68 year-old woman presented with only breast and chest wall mass without renal function damage. It must be stressed that this case is very rare, and it presented with this rare symptom may be related to the infiltration of plasma cells into the corresponding organs. ${ }^{15,16}$ Literature has shown that decreased expression of CD56 may be more likely to occur extramedullary dissemination. ${ }^{14}$

Meanwhile, the imaging manifestations of MM involving breast or superficial soft tissue alone have only been reported in individual cases. ${ }^{17}$ Due to the same pathological origin, $\mathrm{MM}$ and primary plasmacytoma of the breast may 

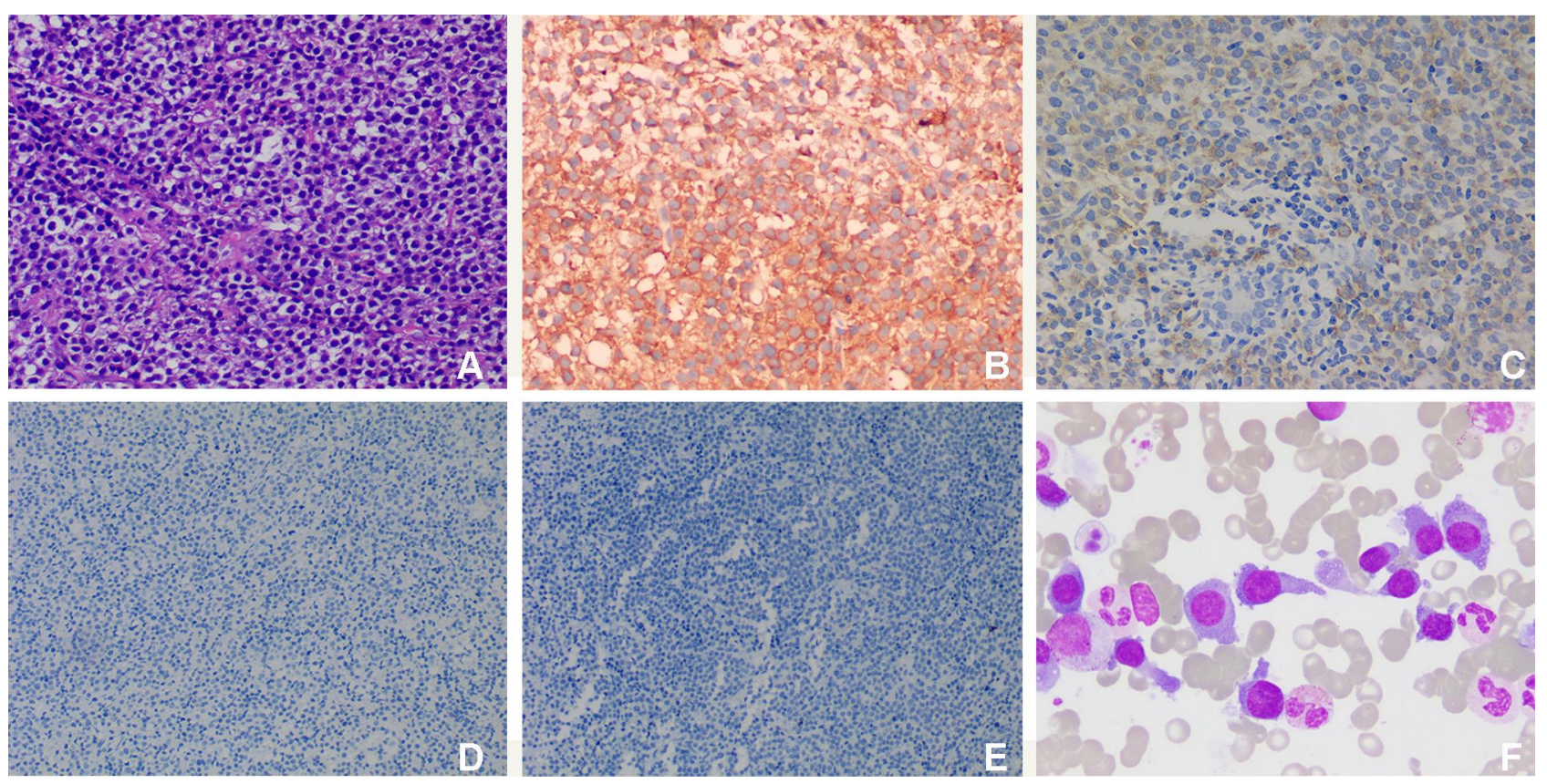

Figure 3 Histopathological examination of masses in the breast and chest wall. (A) HE staining shows lymphocyte proliferation (original magnification, $\times 200$ ); immunohistochemical staining of the proliferated lymphocytes show positive for CDI38 (B, original magnification, $\times 200)$ and IgD (C, original magnification, $\times 200)$, but negative for CD56 (D, original magnification, $\times 100)$ and CDII7 (E, original magnification, $\times 100$ ); (F) bone marrow smears (original magnification, $\times 1000)$ show proplasmocytes and immature plasma cells, which differ in size and shape. The cytoplasm was dark blue with a few granules. The nucleus is biased and binuclear with reticular chromatin and vacuoles in some nuclei and plasma.

have similar imaging manifestations. Surov A reported that MM of breast appeared as a hypoechoic or heterogeneous mass, which may be well-circumscribed and microlobulated, or ill-defined and lobulated. ${ }^{18}$ In addition, microcalcification was extremely rare in MM. ${ }^{19}$ There are various posterior features, and the most common feature is posterior acoustic enhancement, followed by no posterior acoustic transmission, while posterior acoustic shadowing is very rare. In our patient, both the breast and chest wall masses showed posterior enhancement. Some lesions were demonstrated as hypervascular mass with multiple vessels on color Doppler flow imaging, ${ }^{18}$ the breast mass did not have blood flow while the chest wall mass was hypervascular in this case. However, there are few reports about the $\mathrm{CT}$ findings of breast plasmacytoma. ${ }^{20}$ Previous studies on other body parts have indicated that the imaging features of $\mathrm{MM}$ were nonspecific, ${ }^{19}$ most extramedullary plasmacytomas were generally demonstrated as well-defined soft-tissue mass with homogeneous enhancement on CT examination, which was consistent with the breast mass in our patient.

Here, the extramedullary MM presenting as masses on the breast and chest wall complicated with SS was extremely rare. The disappearance of chest wall and breast mass was accompanied by clinical symptoms' relief of SS after treatment for MM, which may indicate the correlation between MM and SS. Further large-scale clinical studies are needed to ascertain whether the coexistence of these two diseases is accidental or intrinsic.

\section{Conclusion}

We reported a unique case of extramedullary MM complicated with SS presenting as breast and chest wall masses, which showed nonspecific imaging characteristics and clinical manifestations. Due attention must be given to patients with simultaneous masses in the superficial organs and tissues, especially in patients with complicated autoimmune diseases such as SS. Ultrasound-guided puncture biopsy is recommended for definite diagnosis to avoid unnecessary misdiagnosis and surgery.

\section{Ethics Approval and Consent}

The study was approved by the ethics committee of West China Hospital, Sichuan University. Written informed consent was received from the patient for publication including the accompanying data.

\section{Acknowledgments}

This work was financially sponsored by the National Natural Science Foundation of China (No. 81671696), the Sichuan Key Research and Development Project (No. 
2019YFS0219) and the Sichuan Science and technology project (No. 2019YJ0710). We thank our laboratory members for their generous help.

\section{Disclosure}

The authors report no conflicts of interest in this work.

\section{References}

1. Michels TC, Petersen KE. Multiple myeloma: diagnosis and treatment. Am Fam Physician. 2017;95(6):373-384.

2. Rajkumar SV, Kumar S. Multiple myeloma: diagnosis and treatment. Mayo Clin Proc. 2016;91(1):101-119. doi:10.1016/j.mayocp.201 5.11.007

3. Terpos E, Angelopoulou MK, Variami E, Meletis JC, Vaiopoulos G. Sjögren's syndrome associated with multiple myeloma. Ann Hematol. 2000;79(8):449-451. doi:10.1007/s002770000166

4. Tazi I, Rachid M, Benchekroun S. Sjogren's syndrome associated with multiple myeloma. Ann Hematol. 2008;79(8):449-451.

5. Sanap R, Athalye A, Madon P, et al. Detection of $\mathrm{t}(14 ; 16)(\mathrm{q} 32 ; \mathrm{q} 22)$ and monosomy 13 by FISH analysis in a patient with multiple myeloma associated with Sjögren's syndrome: the first case report from India. Case Rep Genet. 2013;2013:279801. doi:10.1155/2013/279801

6. Kehrer M, Koob S, Strauss A, Wirtz D, Schmolders J. [Multiple myeloma - current status in diagnostic testing and therapy]. Z Orthop Unfall. 2017;155(5):575-586. doi:10.1055/s-0043-110224. German.

7. Kyle RA, Gertz MA, Witzig TE, et al. Review of 1027 patients with newly diagnosed multiple myeloma. Mayo Clin Proc. 2003;78 (1):21-33. doi:10.4065/78.1.21

8. Shiboski CH, Shiboski SC, Seror R, et al. 2016 American College of Rheumatology/European League against rheumatism classification criteria for primary Sjogren's syndrome: a consensus and data-driven methodology involving three international patient cohorts. Arthritis Rheum. 2017;69(1):35-45. doi:10.1002/art.39859

9. Ambrus J, Suresh L, Peck A. Multiple roles for B-Lymphocytes in Sjogren's syndrome. J Clin Med. 2016;5(10):87. doi:10.3390/ jem5100087
10. Nocturne G, Mariette X. Sjögren syndrome-associated lymphomas: an update on pathogenesis and management. $\mathrm{Br} J$ Haematol. 2015;168(3):317-327. doi:10.1111/bjh.13192

11. Kaneko H, Ohkawara Y, Taniguchi K, et al. Simultaneous complication of multiple myeloma with Sjogren syndrome. Asian Pac $J$ Allergy Immunol. 2006;24(4):245-248.

12. McShane C, Murray L, Landgren O, et al. Prior autoimmune disease and risk of monoclonal gammopathy of undetermined significance and multiple myeloma: a systematic review. Cancer Epidemiol Biomarkers Prev. 2014;23(2):332-342. doi:10.1158/1055-9965.EPI13-0695

13. Zagouri F, Kastritis E, Symeonidis AS, et al. Immunoglobulin D myeloma: clinical features and outcome in the era of novel agents. Eur J Haematol. 2014;92(4):308-312. doi:10.1111/ejh.12255

14. Weinstock M, Ghobrial I. Extramedullary multiple myeloma. Leuk Lymphoma. 2013;54(6):1135-1141. doi:10.3109/10428194.2012.74 0562

15. Gupta N, Rao S, Agarwal P. Multiple myeloma presenting as cryoglobulinaemic vasculitis. $\mathrm{Br} \quad J$ Haematol. 2019;185(5):818. doi:10.1111/bjh. 15868

16. Eslick R, Talaulikar D. Multiple myeloma: from diagnosis to treatment. Aust Fam Physician. 2013;42(10):684-688.

17. Ali H, Nasir Z, Marzouk A. Multiple myeloma breast involvement: a case report. Case Rep Radiol. 2019;2019:2079439. doi:10.1155/ 2019/2079439

18. Surov A, Holzhausen HJ, Ruschke K, Arnold D, Spielmann RP. Breast plasmacytoma. Acta Radiol. 2010;51(5):498-504. doi:10.31 09/02841851003712924

19. Urano M, Denewar FA, Okabe R, et al. Relapsed multiple myeloma manifesting as extramedullary plasmacytoma of the breast: imaging findings. Radiol Case Rep. 2019;14(5):584-587. doi:10.1016/j. radcr.2019.02.018

20. Lee HS, Kim JY, Kang CS, Kim SH, Kang JH. Imaging features of bilateral breast plasmacytoma as unusual initial presentation of multiple myeloma: case report and literature review. Acta Radiol Short Rep. 2014;3(10):1-4. doi:10.1177/2047981614557666
International Journal of General Medicine

\section{Publish your work in this journal}

The International Journal of General Medicine is an international, peer-reviewed open-access journal that focuses on general and internal medicine, pathogenesis, epidemiology, diagnosis, monitoring and treatment protocols. The journal is characterized by the rapid reporting of reviews, original research and clinical studies across all disease areas. The manuscript management system is completely online and includes a very quick and fair peer-review system, which is all easy to use. Visit http://www.dovepress.com/ testimonials.php to read real quotes from published authors. 